\title{
Numerical modelling of the effect of operating parameters in the plastic blown film process
}

\author{
A. Khan* J. J. Shepherd ${ }^{\dagger} \quad$ S. Bhattacharya ${ }^{\ddagger}$
}

(Received 20 December 2004, revised 24 October 2005)

\begin{abstract}
The blown film process with polymer melts is modelled using nonisothermal viscoelastic rheological constitutive equations that are suitable for polyolefins. The model correlates the operating parameters such as mass flows, extruder temperature, tensile axial force and takeup force on processes such as bubble geometry and bubble temperature profile. Unlike Luo and Tanner [Poly. Eng. Sci., Vol. 25, 1985] who used the Maxwell constitutive equation, this study considers the viscoelastic Kelvin model that avoids any assumption regarding stress at the die exit. Like Luo and Tanner, the model uses shooting techniques
\end{abstract}

*School of Civil and Chemical Engineering, RMIT University, Melbourne, Australia. mailto:ashfaq.khan@rmit.edu.au

${ }^{\dagger}$ School of Mathematical and Geospatial Sciences, RMIT University, Melbourne, Australia. mailto: jshep@rmit.edu.au

${ }^{\ddagger}$ School of Civil and Chemical Engineering, RMIT University, Melbourne, Australia. mailto: satinath. bhattacharya@rmit.edu. au

See http://anziamj.austms.org.au/V46/CTAC2004/Kha2 for this article, (c) Austral. Mathematical Soc. 2005. Published November 17, 2005. ISSN 1446-8735 
to match the initial and boundary conditions from the freeze line and the die. The pressure drop and the take-up force are estimated as parameters that are optimized with the boundary conditions using the Nelder-Mead optimization method with Matlab. Effects of varying elasticity parameters and heat transfer coefficients on the bubble geometry, the pressure drop and take-up force are investigated.

\section{Contents}

1 Introduction

C1240

2 Governing equations

$\mathrm{C} 1242$

3 Numerical solution

C1245

4 Results of the simulation

C1246

4.1 Zero shear modulus . . . . . . . . . . . . . . . . . C1246

4.2 Heat transfer coefficient . . . . . . . . . . . . C1247

5 Discussion

C1247

References

C1252

\section{Introduction}

The blown film process is widely used for the commercial production of biaxially oriented thin polymer films that are typically used in the food and packaging industry. These films have desirable physical properties such as tensile strength, low gas permeability and improved tear strength. However, the underlying science is still unclear and thus it is desirable for the plastics 
industry to seek to more clearly understand the physical phenomena associated with this process. One way to do this is to carry out a theoretical modelling of the film blowing process, that examines the complex non-isothermal and non-uniform biaxial extensional processes arising in the interaction between rheology, heat transfer and aerodynamics [3]. Modelling provides a means to control the bubble shape and temperature profile of the bubble by means of changing parameters like bubble pressure and take-up force. Additionally, process modelling also demonstrates how material parameters such as elasticity in the constitutive equation affect the film blowing process.

In this study, a non-isothermal Kelvin model is used to model the blown film process. This model has been used before by Muke et al. [8]. However, in this study a different numerical algorithm is used that optimizes the pressure and take-up force parameters based on the boundary conditions. Like Luo and Tanner [7] this study depends on the assumption of specification of the freezeline; and thus it has an advantage over the Maxwell model used by [7] and Cain and Denn [2], since the stresses at the die exit do not have to be specified.

The initial work of Pearson and Petrie [12] established an understanding of the blown film process, in a classic work in two parts. This work assumes steady-state isothermal Newtonian flows, and has led to a set of graphs that are still used by other authors [10] to analyze the film blowing process. Viscoelastic effects were introduced in [12], by using the original Oldroyd constitutive equations and assuming that properties were independent of temperature. This work found difficulties in achieving numerical answers. This approach was later generalised in [13], by adopting the full energy equation with approximated heat transfer equations.

Much of the work that followed differed in the use of constitutive equation used for polymer melts. Wagner [15] investigated the non-isothermal Maxwell fluid. However this work had to alter the relaxation time and the zero shear viscosity in order to predict the correct bubble profile. The non-isothermal power law model was examined by Han and Park [5], and reasonable agree- 
ment with the experimentally observed radius and film thickness profiles was achieved. Note that recently the model presented in [5] was used in [6] for polethylene. Gupta et al. [4] used the White-Metzner equation of state and published a set of experimental results with full details with polystyrene. This work experienced problems in predicting the circumferential stress.

Luo and Tanner [7] and Cain and Denn [2] used the upper convected Maxwell and Leonov models for modeling of polystyrene, based on the data in [4]. This found that the Leonov model gave poor agreement and that the Maxwell model predicted the data reasonably well. However, both experienced numerical difficulties in solving the defining equations for this model. Cao and Campbell [1] also used the upper Maxwell model with an additional assumption of two phases near the freeze line, that after freezing turned to an elastic solid. They also experienced numerical difficulties. Muke et al. [8] used the non-isothermal Kelvin model with fair success with rheological data on polypropylene. However, it was found that the model has numerical stability problems under some conditions.

Recent work [9] applied the Phan-Thien Tanner (PTT) model to the blown film process. This PTT model is complex and had been modified to include crystallization effects. They could not achieve convergence with the full geometry, so the approach was simplified to a quasi-cylindrical geometry. They claim reasonable agreement with the literature.

\section{Governing equations}

The geometry of the film blowing process is well known and, for example, is described by Shepherd and Bennett [14]. Molten polymer extrudes through an annular die, of radius $a_{0}$ and thickness $H_{0}$, and draws up to form a bubble, maintained by imposed internal air pressure. Under assumptions of axial symmetry, two key variables describe the ultimate bubble shape: the bubble radius $a$, and the film thickness $H$; both variables are functions of the axial 
distance $Z$ from the die exit.

The application of the Voight or Kelvin model was first adopted for film blowing by Pearson and Gutteridge [11]. This model leads more easily to solutions than does the Maxwell model. This has been supported by the modelling of [7], who supply further details of the derivation.

Following [7], dimensionless bubble radius $r$, film thickness $h$ and axial distance $z$ are defined by

$$
r=\frac{a}{a_{0}}, \quad h=\frac{H}{H_{0}}, \quad z=\frac{Z}{a_{0}},
$$

respectively, where $a, H$ and $Z$ are the dimensional distances with the subscript zero indicating the die location. Similarly, dimensionless axial velocity $u$ and principal stresses $S$ and $T$ are

$$
u=\frac{V}{V_{0}}, \quad S=\frac{\sigma_{11} a_{0}}{\eta_{0} V_{0}}, \quad T=\frac{\sigma_{33} a_{0}}{\eta_{0} V_{0}},
$$

where $V, \sigma_{11}, \sigma_{33}$ and $\eta_{0}$ are dimensional axial velocity, principal stresses in the axial and transverse directions and the zero shear viscosity, respectively, with $\eta_{0}$ being, in general, a function of temperature.

The principal stresses $S$ and $T$, in axial and transverse directions respectively, are in the Kelvin form

$$
\begin{aligned}
S & =\alpha \ln \left(\frac{1}{r^{2} h^{4}}\right)-\frac{2 \beta}{r h \sqrt{1+\left(r^{\prime}\right)^{2}}}\left(\frac{2 h^{\prime}}{h}+\frac{r^{\prime}}{r}\right), \\
\text { and } \quad T & =\alpha \ln \left(\frac{r}{h}\right)^{2}+\frac{2 \beta}{r h \sqrt{1+\left(r^{\prime}\right)^{2}}}\left(\frac{r^{\prime}}{r}-\frac{h^{\prime}}{h}\right) .
\end{aligned}
$$

These are the derived forms of the constitutive equations for the present problem; with primes denoting derivatives taken with respect to the axial variable $z$. 
Combination of axial force balance and the pressure with the transverse force leads to the governing equations

$$
\begin{aligned}
& r^{\prime \prime}=\frac{h T \sqrt{1+\left(r^{\prime}\right)^{2}}-2 r B\left(1+\left(r^{\prime}\right)^{2}\right)}{A+B r^{2}} \\
& h^{\prime}=\frac{h}{2}\left[-\frac{r^{\prime}}{r}-\frac{r h \sqrt{1+\left(r^{\prime}\right)^{2}}}{2 \beta}\left(\alpha \ln \left(r^{2} h^{4}\right)+\frac{\left(A+B r^{2}\right) \sqrt{1+\left(r^{\prime}\right)^{2}}}{r h}\right)\right]
\end{aligned}
$$

where $A, B, \alpha$ and $\beta$ are the dimensionless take-up force, bubble pressure, zero shear modulus and zero shear viscosity, respectively.

A more detailed discussion may be found in [8]. Note that the second equation in [8] contained a typographical error, corrected here.

Also note that $\alpha$ and $\beta$ are both functions of dimensionless temperature $t$, with $T_{a}(1+t)$ giving dimensional temperature in terms of $T_{a}$, the ambient temperature $\left(298^{\circ} \mathrm{K}\right.$ here $)$. The coefficient $\alpha$ has a linear dependence

$$
\alpha=a_{10}\left(x_{1}+x_{2} T_{a}(1+t)\right),
$$

whereas the viscosity function, $\beta$, obeys the Arrhenius form

$$
\begin{aligned}
\beta=\exp & {\left[c-a_{1} T_{a}\left(t-t_{0}\right)+\frac{a_{2}}{T_{a}}\left(\frac{1}{1+t}-\frac{1}{1+t_{0}}\right)\right.} \\
+ & \left.a_{3}\left(\left(\frac{1}{T_{a}\left(t-t_{c}\right)}\right)^{a_{4}}-\left(\frac{1}{T_{a}\left(t_{0}-t_{c}\right)}\right)^{a_{4}}\right)\right],
\end{aligned}
$$

where the constants $a_{10}, x_{1}$ and $x_{2}$ are derived from the dynamic shear rheological tests, while the constants $a_{1}, a_{2}, a_{3}$ and $a_{4}$ are determined from the zero shear tests as functions of temperature. The temperature $t_{c}$ is the crystallization temperature.

In addition to the above, the energy equation, as specified in [7] is

$$
t^{\prime}=C_{e}\left[S \frac{r^{\prime}}{r}-T\left(\frac{h^{\prime}}{h}+\frac{r^{\prime}}{r}\right)\right]-C_{h} r t \sqrt{1+\left(r^{\prime}\right)^{2}},
$$


where $C_{e}$ and $C_{h}$ are the dimensionless energy dissipation coefficient and the dimensionless heat transfer coefficient respectively, whereas $t$ is the dimensionless temperature, defined earlier. The boundary conditions are

$$
r(0)=1, \quad h(0)=1,
$$

where $z=0$ locates the die exit. Further specifications at the freezeline height are

$$
r^{\prime}\left(z_{f}\right)=0, \quad r\left(z_{f}\right)=r_{f}, \quad h\left(z_{f}\right)=h_{f} \quad \text { and } \quad t\left(z_{f}\right)=t_{f},
$$

where $z_{f}=Z_{f} / a_{0}$ locates the freezeline.

\section{Numerical solution}

Equations (5), (6) and (9) were solved by starting at the freezeline and numerically integrating back to the die-exit. The second order differential equation (5) for $r$ requires two boundary conditions to be specified (for $r$ and $r^{\prime}$ ); while Equation (6) requires a boundary condition for $h$. Similarly, the first order differential equation (9) needs a specified value for $t$ at one boundary. These requirements are all provided by the boundary conditions (11). However, the die-exit conditions (10) must be met, to make the problem realistic. This is accomplished by adjusting the values of the parameters $A$ and $B$ that govern the axial force acting, and the pressure difference in the bubble.

The integration of the differential equations (5), (6) and (9) subject to (11) was accomplished using the fourth order Runge-Kutta method in Matlab, ode45. The solution commenced at the freezeline $z_{f}$ and terminated at the die-exit $z=0$. At the die-exit, the radius $r$, the profile slope $r^{\prime}$, the thickness $h$ and the temperature $t$ were evaluated. To meet the constraints (10), the shooting method was implemented backwards from the freezeline, such that a least square error

$$
E=(r-1)^{2}+(h-1)^{2},
$$


was minimized at the die-exit, by adjusting the values of $A$ and $B$. This was accomplished by application of the Nelder-Mead algorithm in the Optimization toolbox of Matlab. This algorithm changes $A$ and $B$ and re-solves the shooting problem until the minimal error $E$ as given by (12) is achieved at the die-exit.

Note that for each freezeline height $z_{f}$, the problem is tightly constrained to yield unique values for $A$ and $B$. It would be possible to rearrange the problem to yield $z_{f}$ and $A$ if, for example, $B$ were given.

Note this method assumes the freezeline value. Examination of the recent literature [9] that calculates the freezeline reveals that the pressure value has to be assumed. So in order to calculate the freezeline 'naturally' the value of pressure drop has to be known. In the present study the pressure drop could not be measured and therefore the standard approach was used.

\section{Results of the simulation}

Two parameters of interest have been varied: these are (i) the $x_{1}$ parameter in the shear modulus; and (ii) the dimensionless heat transfer coefficient $C_{h}$. The first illustrates the effects of varying elasticity on the results; and the second shows the effects of the increasing the heat transfer rate as would be caused, for example, by increasing the cooling air flow.

\subsection{Zero shear modulus}

The zero shear modulus was increased by increasing the value of the parameter $x_{1}$ as shown in Table 1 and the outputs of the axial take-up force $A$ and the pressure drop $B$ were included. 
TABLE 1: Values of $A$ and $B$ for increasing zero shear modulus $x_{1}$

\begin{tabular}{|ccc|}
\hline$x_{1}$ & $A$ & $B$ \\
\hline 1843 & 0.7250 & 0.2599 \\
3600 & 0.8819 & 0.3107 \\
7600 & 1.2122 & 0.4170 \\
\hline
\end{tabular}

TABle 2: Variation of $A$ and $B$ with $C_{h}$.

\begin{tabular}{|ccc|}
\hline$C_{h}$ & $A$ & $B$ \\
\hline 0.0096 & 0.725 & 0.260 \\
0.0150 & 0.571 & 0.204 \\
\hline
\end{tabular}

The effect of increase in $x_{1}$ on the radial profile is illustrated in Figure 1.

\subsection{Heat transfer coefficient}

The effects of variation of the heat transfer coefficient on the bubble radius was investigated. Table 2 gives the values of $C_{h}$. The effect of an increase in $C_{h}$ on the radial profile is then illustrated in Figure 2.

\section{Discussion}

This paper may be viewed as an exercise in the modelling of a complex process (the film film blowing of a Kelvin fluid) in order to investigate the influence of the variation of key parameters on that process. In the present case, this variation comprises changes in elasticity and cooling effects, that are effected by varying the parameters $x_{1}$ and $C_{h}$ respectively. The Kelvin model fluid was selected as it is one of the simplest viscoelastic fluids into 


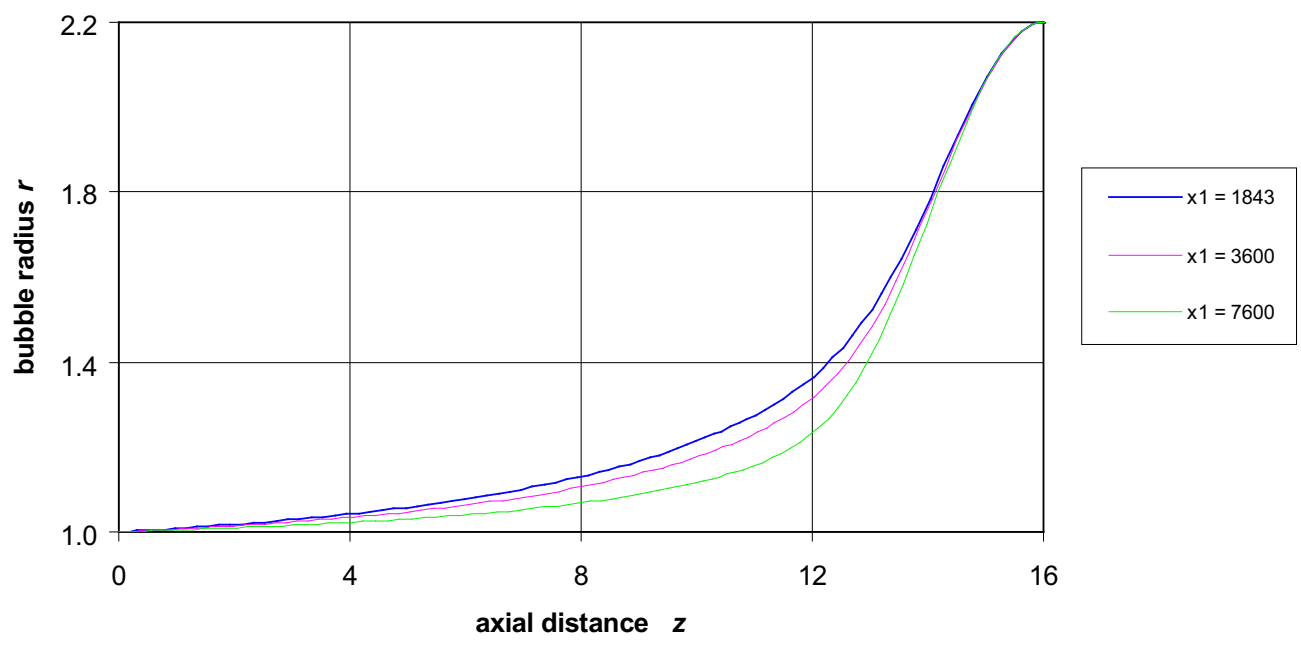

FiguRE 1: Variation of bubble radius profile with $x_{1}$. 


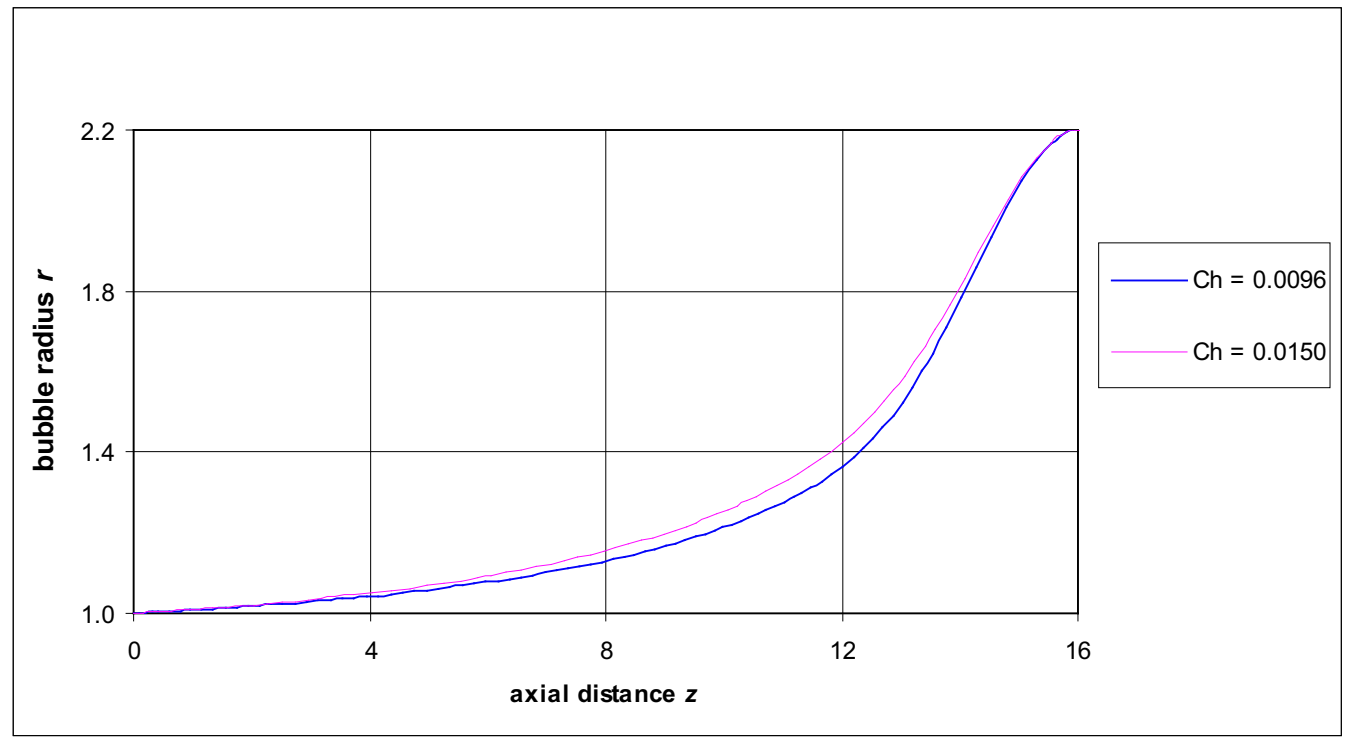

Figure 2: Variation of bubble radius profile with $C_{h}$. 
which thermal effects can be incorporated. The Kelvin constitutive equation, along with that for the Maxwell model, form the simplest ways to represent viscoelastic behaviour in a polymer. While the Maxwell model has been investigated thoroughly and shown to give unstable results that are strongly dependent on the initial conditions at the die, the Kelvin model in the present form has only recently been employed [8]; and, as noted, also incorporates elasticity effects. Because of their coiled molecules, polymers display strong elastic effects and this model does give scope to address some of the questions regarding the influence of elasticity on the ultimate film bubble profile. Figure 1 shows that increasing $x_{1}$, which represents increasing elasticity leads to an increased tendency to necking behaviour in the film bubble; that is, a delayed bubble expansion (eventuating, in cases, in a narrowing of the bubble radius before ultimate expansion). This indicates a tendency towards more solid-like behaviour .

Variations in the convective cooling effect is represented by $C_{h}$, an effective dimensionless convective heat transfer coefficient. $C_{h}$, which is an important process parameter, and the effect of its variation on the bubble profile, can be seen in Figure 2. This shows that increasing $C_{h}$ decreases the tendency to neck; that is, it decreases the slope at the transition point of the profile. This is an interesting phenomenon, since it may well be argued that increasing cooling rate should give a greater tendency towards a more solid like behaviour, resulting in the opposite effect. However, remember that the solution generated here is subject to very tight boundary conditions and this displays a consequence of this. Thus when the cooling rate is increased, by increasing $C_{h}$, the system still is forced to maintain the freeze line height specified; and the only way this may be accomplished is by decreasing the bubble surface area, since the overall cooling load has to be the same. This is accomplished by a reduction in the slope in the bubble radius profile. This demonstrates the unique aspect of this model, in that the energy balance has to be maintained within the very tight boundary conditions.

The variation of $x_{1}$ and $C_{h}$ do not give huge differences in the overall 
shape of the bubble profile; however, as Table 1 shows, there are substantial difference in the corresponding values of $A$ and $B$, especially when varying the elasticity. This is the consequence of the tight boundary conditions that have to be maintained at both ends of the bubble. The slope has to be zero at $x_{f}$, while, in addition, the radius has to be maintained at a specified setting at both ends. Thus the shape of bubble is maintained within close bounds. Overall, we have shown that the how the changes in two important properties have on the overall shape bubble shape.

The only degrees of freedom available to the model are an adjustment of $A$ and $B$, which are related to the axial tensile force and the internal bubble pressure, respectively; in order to satisfy the given boundary conditions. This mimics the degree of freedom that is give to the process operator in real-world film blowing. Usually, the freeze line height is fixed, by the plant geometry. The operator will adjust the air pressure in the bubble (adjust $B$ ) to provide the required blow-up radius; and tension provided by the pull (adjust $A$ ) to give the required film thickness. Since, on a pilot scale, the bubble pressure and the tensile force are not measured quantities, these parameters have been chosen here to be some of the outputs of the modelling process.

This modelling exercise is regarded as valuable as it illustrates the solution of the real-world problem of film blowing that must meet tight constraints. The model is naturally an approximation to the real-world problem; and to achieve a 'quick' solution, both the heat transfer and the constitutional equations have to be grossly simplified. It is suggested that a future activity would be to have the freeze line height (FLH) as an output of the model, while specifying the pressure drop parameter $B$ as an input. This models the operator adding air to the bubble to achieve a specified pressure and subsequently letting the FLH reach its 'natural' setting that satisfies the equations. The specification of the FLH poses a strong constraint on the modelling and it seems likely that if the FLH was allowed to vary, a greater stable window of solution would result. Naturally, other future activities would include better constitutive equations and heat transfer modelling to 
include two-dimensional effects in the film cooling.

\section{References}

[1] B. Cao and G. A. Campbell. Viscoplastic-elastic modeling of the tubular blown film processing. AIChE J., Vol. 36, p.420, 1990. C1242

[2] J. J. Cain and M. M. Denn. Multiplicities and instabilities in film blowing. Poly. Eng. Sci., Vol. 28, p.1527, 1988. C1241, C1242

[3] A. Ghaneh-Fard, P. J. Carreau and P. G. Lafleur. Study of kinematics and dynamics of film blowing of different polyethylenes. Poly. Eng. Sci., Vol. 37, p.1148, 1988. C1241

[4] R. K. Gupta, A. B. Metzner and K. F. Wissbrun. Modeling of polymeric film blowing process. Poly. Eng. Sci., Vol. 22, p.172, 1982. C1242

[5] C. D. Han and J. Y. Park. Studies on blown film extrusion 2. analysis of the deformation and heat transfer processes. J. Appl. Polym. Sci., Vol. 19, p.3277, 1975.

http://dx.doi.org/10.1002/app.1975.070191213 C1241, C1242

[6] H. A. Khonakdar, J. Morsshedian and A. O. Nodehl. Mathematical and computational modeling of heat transfer and deformation in film blowing process. J. Appl. Polym. Sci., Vol. 86, p.2112, 2002. http://dx.doi.org/10.1002/app.10769 C1242

[7] X-L. Luo and R. I. Tanner. A computer study of film blowing. Poly. Eng. Sci., Vol. 25, 1985. C1241, C1242, C1243, C1244

[8] S. Muke, H. Connell, I. Sbarski and S. Bhattacharya. Numerical modelling and experimental verification of blown film processing. 
J. Non-Newt. Fluid Mech., Vol. 116, p.113, 2003.

http://dx.doi.org/10.1016/j.jnnfm.2003.09.002 C1241, C1242, C1244, C1250

[9] I. Muslet and M. Kamal. Computer simulation of the film blowing process incorporating crystallization and viscoelasticity. J. Rheol., Vol. 48, p.525, 2004. http://dx.doi.org/10.1122/1.1718500 C1242, C1246

[10] T. A. Osswald. Polymer processing fundamentals. Hanser, 1998. C1241

[11] J. R. Pearson and P. A. Gutteridge. Stretching flow for thin film production. Part 1. Bubble blowing in the solid phase. J. Non-Newt. Fluid Mech., Vol. 4, p.57, 1978. C1243

[12] J. R. A. Pearson and C. J. S. Petrie. The flow of a tubular film, Part 1. J. Fluid. Mech, Vol. 40, p.1, 1970. C1241

[13] C. J. S. Petrie. Memory effects in non-uniform flow: a study of the behaviour of a tubular film of viscoelastic fluid. Rheologica Acta, Vol. 12, p.92, 1973. C1241

[14] J. J. Shepherd and J. C. Bennett. Interior layer structure in the Newtonian blown film. ANZIAM J., 46(E) pp.C839-C853, 2005. http://anziamj.austms.org.au/V46/CTAC2004/Shep C1242

[15] M. H. Wagner. Das folienblasverfahren als rheologisch-thermodynamischer Prozeß. Rheol. Acta, Vol. 15, p.40, 1976. C1241 